\section{Polygon patterns on Europa}

PIERI $^{1}$, in his study of the lineament and polygon patterns on Europa, has discussed the problem of the development of crack networks in cooling basalt flows and similar planar, contracting systems. $\mathrm{He}$ observed that although an ideal hexagonal pattern maximizes the reduction of strain energy per unit surface area and is therefore the most efficient pattern given an isotropic stress field, nevertheless, even well developed columnar basalts tend to show many pentagons along with the expected hexagons.

The observations by Beard ${ }^{2}$ on basalt flows the data used by Pieri) certainly show a large proportion of pentagons, ranging from $50 \%$ at Mount Rodeix to $35 \%$ at the Giants Causeway. Beard also recorded the mean number of sides of the basalt polygons and obtained values ranging from 5.23 at Mount Rodeix to 5.66 at the Giants Causeway. In an ideal system, with only 3 -rayed crack vertices the mean side number should be six. A simple Monte Carlo model of a contracting cooling basalt can explain the significant occurrence of non-hexagons and also the reason why Beard's side counts were less than the ideal six.

In natural conditions, in an essentially unbounded basalt flow, one would expect the crack pattern to begin forming in a random manner; conditions can never be ideal enough for the entire pattern to develop in one cracking operation. Thus it can be expected to grow from randomly distributed stress centres ${ }^{3}$. Using a simple random number placement method and an effectively infinite field crack pattern, models can be produced which show many of the features observed by Beard. The model networks have a significant number of pentagons and heptagons and with just the random condition operating always have a mean polygon side number of six. However, a small number of extremely short sides are always produced and if these are eliminated the mean side value drops to $\sim 5.6-5.7$.

This suggests that Beard was unable to detect the very shortest sides and that this accounts for his mean side values in the 5.6 region. If this is so then a flow like the Giants Causeway is very close to an ideal flow; the ideal flow has a mean side number of six but as nature favours random processes we should expect a reasonable proportion of pentagons and heptagons. Pieri stated that the more isotropic and uniform the stress, the more likely it will be that hexagons form: this is looking to an ideal-ideal situation; in a real-ideal situation (a possible geological happening) it is likely that the mean side number will approach six but we should still expect non-hexagons to appear in the network.

\section{DSIR Soil Bureau, \\ Lower Hutt, \\ New Zealand \\ 1. Pieri, D. C. Nature 289, 17-21 (1981). \\ 2. Beard, C. N. Bull. geol. Soc. Am. 70, 379-381 (1959) 3. Smalley, I. J. Geol. Mag. 103, 110-114 (1966).}

I. J. SMAlley

PIERI replies-It is clear from Smalley's comment above and from previous work ${ }^{1}$ that polygonal fracture patterns forming in real materials in even the most ideal of isotropic stress regimes are subject to compositional anisotropies at many scales and a fracture network cannot form all at once. Thus, the ideal perfectly-formed pure-hexagonal pattern will never form in nature and will have particular difficulty in the context of global planetary fracture patterns ${ }^{2}$. I did not mean to imply that the end result of optimized real conditions would be pure hexagonal patterns, but rather that hexagons would be more numerous in situations where conditions were more uniform. Clearly, pentagons and heptagons will still be present, and I thank Smalley for clarifying this point.

The research described in this note was carried out by the Jet Propulsion Laboratory, California Institute of Technology, under contract with NASA.

\section{C. PIERI}

Jet Propulsion Laboratory,

California Institute of

Technology,

Pasadena, California 91109, USA

1. Smalley, I. J. Geol. Mag. 10, 110-114 (1966)

2. Pieri, D. C. Nature 289, 17-21 (1981).

\section{Low-angle X-ray scattering of chromatin}

LANGMORE AND SCHUTT ${ }^{1}$ have recently reported low-angle $\mathrm{X}$-ray scattering experiments on chicken erythrocytes in which they find a peak at $\sim 400 \AA$ in the plot of $s^{2} I$ against $s$. They conclude that this peak is related to the side-by-side packing of chromosome fibres. I wish to show here that this peak may have a different interpretation.

Langmore and Schutt $^{1}$ multiply the recorded intensity $I$ by $s^{2}$ to correct for the random orientation of the fibres with respect to the X-ray beam, where $s=2$ $\sin (\theta / 2) / \lambda$. However, as shown quite generally by Porod $^{2}$ and discussed in detail by Mittelbach ${ }^{3}$, information on the cross-section properties of rod-like parti- cles is best obtained from the study of the dependence of $I s$ (instead of $I s^{2}$ ) on $s$.

At very low angles, a system of rod-like particles obeys the approximate Guinier equation:

$$
(I s)=K \exp \left[-2\left(\pi s R_{\mathrm{G}}\right)^{2}\right]
$$

where $K$ is a constant and $R_{\mathrm{G}}$ the radius of gyration of the cross-section. For a cylindrical particle of uniform density and radius $R, R_{\mathrm{G}}=0.707 R$. If $I s^{2}$ is plotted instead of $I s$, a maximum in the curve will appear when $\mathrm{d}\left(I s^{2}\right) / \mathrm{d} s=0$. Introducing this condition in equation (1), it is easily shown that a maximum in the plot of $I s^{2}$ against $s$ should appear for

$$
s=1 / 2 \pi R_{\mathrm{G}}
$$

Therefore, the maximum found by Langmore and Schutt ${ }^{1}$ in the plots of $I s^{2}$ against $s$ for $s=1 / 400 \AA^{-1}$ is consistent with the presence of cylindrical objects with a cross-sectional radius of gyration $R_{\mathrm{G}}=64 \AA$, as calculated from equation (2). This value would correspond to cylindrical particles of 180 - $\AA$ diameter if their electron density were uniform. Thus I think that the peak observed by Langmore and Schutt ${ }^{1}$ is due to intrinsic features of the chromatin fibres and does not demonstrate any regular side-by-side packing of chromosome fibres.

I suggest that the determination of the position of maxima and the use of equation (2) may be a useful alternative to Guinier plots in determining the value of $\boldsymbol{R}_{\mathrm{G}}$. A similar procedure could be used for globular particles, but in that case the maxima in the plot of $I s$ against $s$ would be used.

JuAN A. SUbirana

Unidad de Quimica Macromolecular,

Consejo Superior de Investigaciones Cientificas,

Escuela Técnica Superior de

Ingenieros Industriales,

Diagonal 999, Barcelona, Spain

1. Langmore, J. P. \& Schutt, C. Nature 288, 620-622 (1980). 2. Porod, G. Acta phys. austriaca 2, 255-292 (1948)

3. Mittelbach, P. Acta phys. austriaca 19, 53-102 (1964)

LANGMORE AND SCHUTT REPLYAlthough it is necessary that widely spaced homogeneous cylindrical fibres of diameter $D$ give rise to a maximum in $s^{2} I$ at $\sim s=(2.2 D \AA)^{-1}$, a peak in the $s^{2} I$ curve is not sufficient to prove the existence of widely spaced homogeneous cylindrical particles. The analysis that Subirana suggested is only relevant to the determination of the radius of gyration of particles provided that these particles are rod-like and widely separated. Proper Guinier analysis ${ }^{1}$ tests the validity of these 